\title{
Polymorphic Uncertain Linear Programming for Generalized Production Planning Problems
}

\author{
Xinbo Zhang, ${ }^{1}$ Feng Zhang, ${ }^{1}$ Xiaohong Chen, ${ }^{2}$ and Zhong Wan ${ }^{1}$ \\ ${ }^{1}$ School of Mathematics and Statistics, Central South University, Changsha, Hunan 410083, China \\ ${ }^{2}$ School of Business, Central South University, Changsha, Hunan 410083, China \\ Correspondence should be addressed to Zhong Wan; wanmath@163.com
}

Received 24 June 2013; Revised 28 December 2013; Accepted 2 January 2014; Published 19 February 2014

Academic Editor: Cheng-Hong Yang

Copyright (c) 2014 Xinbo Zhang et al. This is an open access article distributed under the Creative Commons Attribution License, which permits unrestricted use, distribution, and reproduction in any medium, provided the original work is properly cited.

\begin{abstract}
A polymorphic uncertain linear programming (PULP) model is constructed to formulate a class of generalized production planning problems. In accordance with the practical environment, some factors such as the consumption of raw material, the limitation of resource and the demand of product are incorporated into the model as parameters of interval and fuzzy subsets, respectively. Based on the theory of fuzzy interval program and the modified possibility degree for the order of interval numbers, a deterministic equivalent formulation for this model is derived such that a robust solution for the uncertain optimization problem is obtained. Case study indicates that the constructed model and the proposed solution are useful to search for an optimal production plan for the polymorphic uncertain generalized production planning problems.
\end{abstract}

\section{Introduction}

For a manufacturer, a favorable production planning involves suitably forecasting the demand of products and translating the demand forecast into a plan of production that maximizes the enterprise's profit or minimizes its cost. It has been viewed as a useful approach to formulate the production planning problem into an optimization model by constructing a function of profit or cost as the objective and employing some equalities and inequalities to describe the constraints in the process of production that include the limitations of production resources, the capacity, and the demand of customers.

Actually, in the fields of applied mathematics, operation research, and logistics management sciences, there are many relevant achievements on the problem of production planning. For the recent advancement, for example, see [1-11] and the references therein. It is noted that in the constructed models of production planning, some uncertain factors in the real-world production process, such as the price of product, the customer demand, and the production capacity, are taken into consideration (see [2, 3, 5-11]). For example, in [5], the production capacities and the customer demands are represented by interval numbers, respectively. In $[2,3]$, the customer demands, the prices of products, and the production capacities are described as fuzzy subsets. In [6], random parameters are used to denote the customer demand and the production capacity.

Very recently, it is noted that, in [12], a polymorphic uncertain model is first employed to formulate a class of optimal design problems of belt drive, and an efficient algorithm, called the two-step based sampling algorithm, is developed to maximize the capacity of V-belt driving. Since polymorphic uncertainty also exists in production planning, different from the existent results, this paper intends to construct a polymorphic uncertain model for the generalized production planning problem, where the amount of production, the amount of inventory, and the unmet demand are the decision variables, the customer demands are represented by interval numbers, and the consumption of raw material and the amounts of all resources available are described as fuzzy subsets. Based on the theory of fuzzy interval program 
and the modified possibility degree for the order of interval numbers, we shall convert the new model into a deterministic one and find a robust optimal solution for the original uncertain problem by the standard smooth optimization techniques.

The rest of the paper is organized as follows. In the next section, a polymorphic uncertain linear programming (PULP) model for the generalized production planning problem is constructed. In Section 3, an approach is presented to transform the PULP model into a deterministic equivalent formulation and a solution method is presented. Section 4 is devoted to show the validity of the model and the solution method by case study and sensitivity analysis. Final remarks are given in the last section.

\section{Model of Generalized Production Planning Problem}

In this section, we are going to construct a polymorphic uncertain linear programming (PULP) model for the generalized production planning problem.

We first introduce some denotations.

$T$ : The number of the scheduled production period

I: The number of items (raw materials or finished products)

\section{$K$ : The number of resources}

$a_{i k}$ : The amount of resources $k$ required per unit of product $i$

$b_{k t}$ : The amount of resource $k$ available in period $t$

$d_{i t}$ : The demand for item $i$ in period $t$

$r_{i t}$ : The unit revenue for item $i$ in period $t$

$c p_{i t}$ : The unit variable cost of production for item $i$ in period $t$

$c q_{i t}$ : The unit inventory holding cost for item $i$ in period $t$

$c u_{i t}$ : The unit cost of unmet demand for item $i$ during period $t$

$p_{i t}$ : The production amount of item $i$ during period $t$

$q_{i t}$ : The inventory amount of item $i$ at the end of period $t$

$\mathfrak{R}$ : The set of fuzzy subsets

$R:$ The set of real numbers

$u_{i t}$ : The unmet demand amount of item $i$ during period $t$.
In [5], a deterministic optimization model is presented to formulate the production planning problem:

$$
\begin{array}{ll}
\max & f=\sum_{i=1}^{I} \sum_{t=1}^{T}\left[r_{i t}\left(d_{i t}-u_{i t}\right)-c p_{i t} p_{i t}-c q_{i t} q_{i t}-c u_{i t} u_{i t}\right] \\
\text { s.t. } & \sum_{i=1}^{I} a_{i k} p_{i t} \leq b_{k t}, \quad \forall k, t, \\
& q_{i, t-1}+p_{i t}-q_{i t}-u_{i t}=d_{i t}, \quad \forall i, t, \\
& p_{i t}, q_{i t}, u_{i t} \geq 0, \quad \forall i, t,
\end{array}
$$

where $p_{i t}, q_{i t}$, and $u_{i t}$, for $i=1,2, \ldots, I$ and $t=1,2, \ldots, T$, are the decision variables. In Model (1), the objective function is to maximize the net revenues of production and minimize the cost of inventory and product shortage. The first type of constraints is on the availability of resource such as the labor, the equipment, and the raw material for the production. The second type of constraints is on the material balance. The last type of constraints in Model (1) is on the nonnegativity of decision variables.

Different from an ordinary model of production planning problem, the inventory amount $q_{i t}$ and the unmet demand amount $u_{i t}$ are incorporated into Model (1) as decision variables. Thus, it is called a generalized production planning problem.

However, in the real-world process of production, some uncertain factors should be taken into account before the planing of production. It is often that the demand of customer, the consumption of raw material for each product, and the amount of resource available are time-varying. Furthermore, it is unsuitable if all the uncertainties are described by the same mathematical concept such as the random variable (see [6]). An efficient approach is to incorporate the polymorphic uncertainties existing in the problem into the model, and their mathematical descriptions should reflect the features of the time varying parameters as much as possible. In this connection, one can see [12-14].

We now present a polymorphic uncertain linear programming (PULP) model to formulate the problem of production planning as follows:

$$
\begin{gathered}
\max \quad f^{ \pm}=\sum_{i=1}^{I} \sum_{t=1}^{T}\left[r_{i t}\left(\left[d_{i t}^{-}, d_{i t}^{+}\right]-u_{i t}\right)-c p_{i t} p_{i t}\right. \\
\left.-c q_{i t} q_{i t}-c u_{i t} u_{i t}\right] \\
\text { s.t. } \quad \sum_{i=1}^{I} \tilde{a}_{i k} p_{i t} \leq \tilde{b}_{k t}, \quad k=1,2, \ldots, K, \\
\quad t=1,2, \ldots, T, \\
q_{i, t-1}+p_{i t}+u_{i t}=\left[d_{i t}^{-}, d_{i t}^{+}\right], \quad i=1,2, \ldots, I, \\
p_{i t}, q_{i t}, u_{i t} \geq 0, \quad i=1,2, \ldots I, \quad t=1,2, \ldots, T .
\end{gathered}
$$


Different from (1), in Model (2), the amount of resources $k$ required per unit of production of item $i$, denoted by $\widetilde{a}_{i k}$, are assumed to be a triangle fuzzy subset. Similarly, the amount of resources $k$ available in period $t$, being referred to as $\widetilde{b}_{k t}$, are also a triangle fuzzy subset. The demand of product $i$ in period $t$ is regarded as an interval number, denoted by $\left[d_{i t}^{-}, d_{i t}^{+}\right]$.

Remark 1. When there exist these uncertain parameters in an optimization model, it is impossible to find an optimal solution in the view point of standard optimization theory. However, in virtue of the method proposed in [12] for the optimal design problem of driving system with polymorphic uncertainties, we can determine an approximate solution of Model (2) by transforming Model (2) into a deterministic one for the given degree of satisfaction as shown in the next section.

\section{Deterministic Equivalent Formulation}

In this section, we study the deterministic equivalent formulation for the PULP Model (2), which is useful to find a robust optimal solution for an uncertain problem.

We first address how to convert a fuzzy linear program into an interval one.

Consider the following linear programming problem:

$$
\begin{array}{ll}
\max & f=\sum_{j=1}^{n} c_{j} x_{j} \\
\text { s.t. } & \sum_{j=1}^{n} \tilde{a}_{i j} x_{j} \leq \tilde{b}_{i}, \quad i=1,2, \ldots, m, \\
& x_{j} \geq 0,
\end{array}
$$

where $\tilde{a}_{i j} \in \mathfrak{R}$ and $\tilde{b}_{i} \in \mathfrak{R}$, for $i=1, \ldots, m$ and $j=1, \ldots, n$, are fuzzy subsets with the same types of membership function and $c_{j} \in R$, for $j=1, \ldots, n$, are given constants.

For a fuzzy subset, $\alpha$-cut is fundamental concept to approximate a fuzzy subset for a given satisfactory level $\alpha$. For convenience, we state it as follows.

Definition 2 ( $\alpha$-cut). Let $\widetilde{A}$ be a fuzzy subset in the domain $X . \mu_{\widetilde{A}}$ is its membership function. For $0<\alpha \leq 1$, the set

$$
\widetilde{A}_{\alpha}=\left\{x \in X \mid \mu_{\widetilde{A}}(x) \geq \alpha\right\}
$$

is called the $\alpha$-cut of $\widetilde{A}$.

By definition, it is clear that if $\mu_{\widetilde{A}}$ is a quasiconcave function, then $\widetilde{A}_{\alpha}$ is an interval. In the case that $\widetilde{A}$ is a trapezoidal fuzzy subset with a membership function given by

$$
\mu_{\widetilde{A}}(x ; a, b, c, d)= \begin{cases}0, & x \leq a, x>d, \\ \frac{x-a}{b-a}, & a<x \leq b, \\ 1, & b<x \leq c, \\ \frac{d-x}{d-c}, & c<x \leq d,\end{cases}
$$

where $a<b<c<d$, then, from (4), it follows that

$$
\widetilde{A}_{\alpha}=[(1-\alpha) a+\alpha b,(1-\alpha) d+\alpha c] .
$$

Throughout this paper, all membership functions are supposed to be quasiconcave. In the following, we present a relation between a fuzzy linear program and an interval one.

From the definition of $\alpha$-cut and the decomposition theorem in the theory of fuzzy mathematics, we know that

$$
\begin{gathered}
\widetilde{b}_{i}=\bigcup_{\alpha \in[0,1]} \alpha\left(\widetilde{b}_{i}\right)_{\alpha}, \\
\sum_{j=1}^{n} \widetilde{a}_{i j} x_{j}=\bigcup_{\alpha \in[0,1]} \alpha\left(\sum_{j=1}^{n}\left(\tilde{a}_{i j}\right)_{\alpha} x_{j}\right) .
\end{gathered}
$$

Thus, the fuzzy inequality

$$
\sum_{j=1}^{n} \tilde{a}_{i j} x_{j} \leq \tilde{b}_{i}
$$

is equivalent to

$$
\bigcup_{\alpha \in[0,1]} \alpha\left(\sum_{j=1}^{n}\left(\tilde{a}_{i j}\right)_{\alpha} x_{j}\right) \leq \bigcup_{\alpha \in[0,1]} \alpha\left(\widetilde{b}_{i}\right)_{\alpha} .
$$

Denote that

$$
\left[a_{i j}^{-}, a_{i j}^{+}\right]=\bigcup_{\alpha \in[0,1]} \alpha\left(\tilde{\alpha}_{i j}\right)_{\alpha}, \quad\left[b_{i}^{-}, b_{i}^{+}\right]=\bigcup_{\alpha \in[0,1]} \alpha\left(\tilde{b}_{i}\right)_{\alpha} .
$$

If $x^{*}$ solves the following interval program:

$$
\begin{array}{ll}
\max & f=\sum_{j=1}^{n} c_{j} x_{j} \\
\text { s.t. } & \sum_{j=1}^{n}\left[a_{i j}^{-}, a_{i j}^{+}\right] x_{j} \leq\left[b_{i}^{-}, b_{i}^{+}\right], \quad i=1,2, \ldots, m, \\
& x_{j} \geq 0, \quad j=1,2, \ldots, n,
\end{array}
$$

then $x^{*}$ is called a solution of Model (3).

Given constants $\alpha_{s} \in(0,1], s=1,2, \ldots, k$, we can obtain approximate intervals of $\left[a_{i j}^{-}, a_{i j}^{+}\right]$and $\left[b_{i}^{-}, b_{i}^{+}\right]$as follows:

$$
\left[a_{i j}^{-}, a_{i j}^{+}\right] \approx \bigcup_{s=1}^{k} \alpha_{s}\left(\widetilde{\alpha}_{i j}\right)_{\alpha_{s}}, \quad\left[b_{i}^{-}, b_{i}^{+}\right] \approx \bigcup_{s=1}^{k} \alpha_{s}\left(\widetilde{b}_{i}\right)_{\alpha_{s}} .
$$

Thus, the fuzzy inequality

$$
\sum_{j=1}^{n} \widetilde{a}_{i j} x_{j} \leq \widetilde{b}_{i}
$$

is approximated by

$$
\sum_{j=1}^{n}\left(\bigcup_{s=1}^{k} \alpha_{s}\left(\widetilde{\alpha}_{i j}\right)_{\alpha_{s}}\right) x_{j} \leq \bigcup_{s=1}^{k} \alpha_{s}\left(\widetilde{b}_{i}\right)_{\alpha_{s}} .
$$


To give a definition of robust solution for a fuzzy program, we are in a position to transform an interval linear program into a standard smooth optimization problem by a method of modified possibility degree.

We first present an axiomatic definition of a possibility degree operator.

Definition 3. Let $\mathscr{I}$ denote the set of all interval numbers, and let $\mathscr{R}^{I}$ denote the set of the relationship between the interval numbers. If a mapping $P: \mathscr{R}^{I} \rightarrow[0,1]$ satisfies the following conditions:

(1) if $A=\left[a^{-}, a^{+}\right], B=\left[b^{-}, b^{+}\right], P(A \leq B)=1$ if and only if $a^{+} \leq b^{-}$

(2) if $A=\left[a^{-}, a^{+}\right], B=\left[b^{-}, b^{+}\right], P(A \leq B)=0$ if and only if $a^{-} \geq b^{+}$

(3) $P(A \leq B)=1-P(B \geq A)$,

(4) $P(A \leq B)=P(A \geq B)$ if and only if $A=B$,

then $P$ is called a possibility degree operator on $\mathscr{R}^{I}$.

By definition, it is easy to prove the following operator to be a possibility degree operator in $\mathscr{R}^{I}$ :

$$
\begin{aligned}
& P\left(\left[a^{-}, a^{+}\right] \leq\left[b^{-}, b^{+}\right]\right) \\
& \quad= \begin{cases}0, & a^{-} \geq b^{+}, \\
0.5 \frac{b^{+}-a^{-}}{a^{+}-a^{-}} \frac{b^{+}-a^{-}}{b^{+}-b^{-}}, & b^{-} \leq a^{-}<b^{+} \leq a^{+}, \\
\frac{b^{-}-a^{-}}{a^{+}-a^{-}}+0.5 \frac{b^{+}-b^{-}}{a^{+}-a^{-}}, & a^{-}<b^{-}<b^{+} \leq a^{+}, \\
\frac{b^{-}-a^{-}}{a^{+}-a^{-}}+\frac{a^{+}-b^{-}}{a^{+}-a^{-}} \frac{b^{+}-a^{+}}{b^{+}-b^{-}} & \\
+0.5 \frac{a^{+}-b^{-}}{a^{+}-a^{-}} \frac{a^{+}-b^{-}}{b^{+}-b^{-}}, & a^{-} \leq b^{-}<a^{+} \leq b^{+}, \\
\frac{b^{+}-a^{+}}{b^{+}-b^{-}}+0.5 \frac{a^{+}-a^{-}}{b^{+}-b^{-}}, & b^{-} \leq a^{-}<a^{+}<b^{+}, \\
1, & a^{+}<b^{-} .\end{cases}
\end{aligned}
$$

Based on the definition of improved interval possibility degree, we are going to prove the following result.

Theorem 4. Let $P$ be a possibility degree operator defined by (15). Let $x^{*}$ be a solution of the following interval programming model:

$$
\begin{array}{ll}
\max & f^{ \pm}=\sum_{j=1}^{n} c_{j}^{ \pm} x_{j} \\
\text { s.t. } & \sum_{j=1}^{n} a_{i j}^{ \pm} x_{j} \leq b_{i}^{ \pm}, \quad i=1,2, \ldots, m, \\
& x_{j} \geq 0, \quad j=1,2, \ldots, n .
\end{array}
$$

Then, for any $\beta \in[0,1]$ and $\lambda \in[0,1], x^{*}$ is a solution of the following smooth optimization problem:

$$
\begin{array}{ll}
\max & f=\lambda f^{-}+(1-\lambda) f^{+}=\lambda \sum_{j=1}^{n} c_{j}^{-} x_{j}+(1-\lambda) \sum_{j=1}^{n} c_{j}^{+} x_{j} \\
\text { s.t. } & P\left(\sum_{j=1}^{n} a_{i j}^{ \pm} x_{j} \leq b_{i}^{ \pm}\right) \geq \beta, \quad i=1,2, \ldots, m, \\
& x_{j} \geq 0, \quad j=1,2, \ldots, n .
\end{array}
$$

Proof. From the definition of possibility degree in (15), the interval constraint conditions in Model (16) can be converted into its deterministic formulation by introducing the possibility degree parameter $\beta$ : that is,

$$
P\left(\sum_{j=1}^{n} a_{i j}^{ \pm} x_{j} \leq b_{i}^{ \pm}\right) \geq \beta, \quad i=1,2, \ldots, m .
$$

Remark 5. For different sizes of $\beta$, there exist 6 cases for evaluating the possibility degree in the following inequality:

$$
P\left(\sum_{j=1}^{n} a_{i j}^{ \pm} x_{j} \leq b_{i}^{ \pm}\right) \geq \beta .
$$

Actually, (i) if $\beta=0$, then it is required that

$$
\sum_{j=1}^{n} a_{i j}^{-} x_{j} \geq b_{i}^{+}, \quad i=1,2, \ldots, m
$$

(ii) if $\beta=1$, then it is needed that

$$
\sum_{j=1}^{n} a_{i j}^{+} x_{j} \leq b_{i}^{-}, \quad i=1,2, \ldots, m
$$

(iii) if $\beta \in(0,1)$, then the cases from 2 to 5 in (15) subsequently occur as an increment of $\beta$. Since there are $m$ interval constraints in Model (16), the obtained ordinary constraint is one of the four cases for each interval constraint. So, we should treat the original interval programming problem as $4^{m}$ submodels. By comparing the behavior of the solution corresponding to each submodel, a suitable $\beta$ and the corresponding solution are determined. For example, we fix a medium value of $\beta$ such that the third case is used to evaluate the possibility degree in (19): then the corresponding submodel turns out to be

$$
\begin{array}{ll}
\max & f=\lambda f^{-}+(1-\lambda) f^{+} \\
\text {s.t. } & \frac{b_{i}^{-}-\sum_{j=1}^{n} a_{i j}^{-} x_{j}}{\sum_{j=1}^{n} a_{i j}^{+} x_{j}-\sum_{j=1}^{n} a_{i j}^{-} x_{j}}
\end{array}
$$




$$
\begin{gathered}
+0.5 \frac{b_{i}^{+}-b_{i}^{-}}{\sum_{j=1}^{n} a_{i j}^{+} x_{j}-\sum_{j=1}^{n} a_{i j}^{-} x_{j}} \geq \beta, \\
i=1,2, \ldots, m, \\
\sum_{j=1}^{n} a_{i j}^{-} x_{j}<b_{i}^{-}<b_{i}^{+} \leq \sum_{j=1}^{n} a_{i j}^{+} x_{j}, \\
i=1,2, \ldots, m, \\
x_{j} \geq 0, \quad j=1,2, \ldots, n .
\end{gathered}
$$

If the level of possibility degree is large enough such that only one case (e.g., case 4 or case 5) needs to be treated and then an optimization problem with interval parameters can be converted into an ordinary linear program. Actually, from (15), it is known that the possibility degree is ascending as $\beta$ increases. Since a decision-maker in real world always hopes a higher possibility degree, we obtain a deterministic equivalent formulation for the fuzzy linear program (3). Then, based on the above discussion, a concept of robust optimal solution for the PULP Model (2) can be defined.

Definition 6. $x^{*} \in R^{n}$ is called a robust optimal solution of the following PULP problem:

$$
\begin{array}{ll}
\max & f^{ \pm}=\sum_{j=1}^{n} c_{j}^{ \pm} x_{j} \\
\text { s.t. } & \sum_{j=1}^{n} a_{i j}^{ \pm} x_{j} \leq b_{i}^{ \pm}, \quad i=1,2, \ldots, k, \\
& \sum_{j=1}^{n} \widetilde{a}_{i j} x_{j} \leq \widetilde{b}_{i}, \quad i=k+1, k+2, \ldots, m, \\
& x_{j} \geq 0, \quad j=1,2, \ldots, n,
\end{array}
$$

if for given $\lambda \in[0,1], \alpha \in(0,1]$, and $\beta \in(0,1)$ large enough, it solves the deterministic optimization problem as below:

$$
\begin{gathered}
\max \quad f=\lambda f^{-}+(1-\lambda) f^{+} \\
\text {s.t. } \quad P\left(\sum_{j=1}^{n} a_{i j}^{ \pm} x_{j} \leq b_{i}^{ \pm}\right) \geq \beta, \\
i=1,2, \ldots, k, \\
P\left(\sum_{j=1}^{n}\left(\widetilde{a}_{i j}\right)_{\alpha} x_{j} \leq\left(\widetilde{b}_{i}\right)_{\alpha}\right) \geq \beta, \\
i=k+1, k+2, \ldots, m, \\
x_{j} \geq 0, \quad j=1,2, \ldots, n,
\end{gathered}
$$

where $\left(\widetilde{a}_{i j}\right)_{\alpha}$ and $\left(\widetilde{b}_{i}\right)_{\alpha}$ denote the $\alpha$-cuts of $\widetilde{a}_{i j}$ and $\widetilde{b}_{i}$, respectively.
In the meaning of Definition 6, Problem (24) is called a deterministic equivalent formulation of (23). The following result is directly from Theorem 4 and Definition 6.

Theorem 7. Suppose that $\widetilde{a}_{i k}=\left(r_{i k 1}, r_{i k 2}, r_{i k 3}\right)$ and $\widetilde{b}_{k t}=$ $\left(e_{k t 1}, e_{k t 2}, e_{k t 3}\right)$ are triangle fuzzy subsets. Then,

(1) for any given $\alpha \in(0,1)$, the cut-sets

$$
\begin{aligned}
& \left(\widetilde{a}_{i k}\right)_{\alpha}=\left[(1-\alpha) r_{i k 1}+\alpha r_{i k 2},(1-\alpha) r_{k t 3}+\alpha r_{k t 2}\right], \\
& \left(\widetilde{b}_{k t}\right)_{\alpha}=\left[(1-\alpha) e_{k t 1}+\alpha e_{k t 2},(1-\alpha) e_{k t 3}+\alpha e_{k t 2}\right] ;
\end{aligned}
$$

(2) the following linear program in the decision variables $p_{i t}, q_{i t}$, and $u_{i t}$ :

$$
\begin{gathered}
\max \quad f=\lambda f^{-}+(1-\lambda) f^{+} \\
\text {s.t. } 0.5\left(\sum_{i=1}^{I}\left[(1-\alpha) r_{i k 3}+\alpha r_{i k 2}\right] p_{i t}\right. \\
\left.-\left[(1-\alpha) e_{k t 1}+\alpha e_{k t 2}\right]\right)^{2} \\
\times\left(\sum_{i=1}^{I}(1-\alpha)\left(r_{i k 3}-r_{i k 1}\right) p_{i t}\right. \\
\left.\quad \times(1-\alpha)\left(e_{k t 3}-e_{k t 1}\right)\right)^{-1} \\
\leq 1-\beta, \quad \forall k, t, \\
\sum_{i=1}^{I}\left[(1-\alpha) r_{i k 1}+\alpha r_{i k 2}\right] p_{i t} \leq\left[(1-\alpha) e_{k t 1}+\alpha e_{k t 2}\right],
\end{gathered}
$$

$\forall k, t$,

$$
\begin{array}{r}
\sum_{i=1}^{I}\left[(1-\alpha) r_{i k 3}+\alpha r_{i k 2}\right] p_{i t} \geq\left[(1-\alpha) e_{k t 1}+\alpha e_{k t 2}\right], \\
\forall k, t, \\
\sum_{i=1}^{I}\left[(1-\alpha) r_{i k 3}+\alpha r_{i k 2}\right] p_{i t} \leq\left[(1-\alpha) e_{k t 3}+\alpha e_{k t 2}\right],
\end{array}
$$

$\forall k, t$

$$
\begin{aligned}
& q_{i, t-1}+p_{i t}+u_{i t} \geq d_{i t}^{-}, \quad \forall i, t, \\
& q_{i, t-1}+p_{i t}+u_{i t} \leq d_{i t}^{+}, \quad \forall i, t,
\end{aligned}
$$

$p_{i t}, q_{i t}, u_{i t} \geq 0, \quad \forall i, t$, 
TABLE 1: Values of $c p_{i t}, c q_{i t}, c u_{i t}, r_{i t}$, and $d_{i t}^{ \pm}$.

\begin{tabular}{lcc}
\hline$c p_{i t} / c q_{i t} / c u_{i t} / r_{i t} / d_{i t}^{ \pm}$ & $t=1$ & $t=2$ \\
\hline$i=1$ & $1 / 1 / 2 / 4 /[2,16]$ & $2 / 2 / 3 / 3 /[2,14]$ \\
$i=2$ & $3 / 3 / 1 / 2 /[2,12]$ & $1 / 1 / 2 / 4 /[5,16]$ \\
$i=3$ & $2 / 1 / 2 / 4 /[3,15]$ & $1 / 1 / 1 / 4 /[4,18]$ \\
$i=4$ & $1 / 1 / 2 / 4 /[2,16]$ & $2 / 2 / 3 / 3 /[2,14]$ \\
$i=5$ & $3 / 3 / 1 / 2 /[2,12]$ & $1 / 1 / 2 / 4 /[5,16]$ \\
\hline
\end{tabular}

TABLE 2: Cuts of $\tilde{a}_{i k}$.

\begin{tabular}{lccccc}
\hline$\widetilde{a}_{i k}$ & $k=1$ & $k=2$ & $k=3$ & $k=4$ & $k=5$ \\
\hline$i=1$ & $(0,3,6)$ & $(1,4,7)$ & $(3,6,9)$ & $(0,3,6)$ & $(1,4,7)$ \\
$i=2$ & $(3,6,9)$ & $(2,5,8)$ & $(5,8,11)$ & $(3,6,9)$ & $(2,5,8)$ \\
$i=3$ & $(1,4,7)$ & $(5,8,11)$ & $(7,10,13)$ & $(1,4,7)$ & $(5,8,11)$ \\
$i=4$ & $(0,3,6)$ & $(1,4,7)$ & $(3,6,9)$ & $(0,3,6)$ & $(1,4,7)$ \\
$i=5$ & $(3,6,9)$ & $(2,5,8)$ & $(5,8,11)$ & $(3,6,9)$ & $(2,5,8)$ \\
\hline
\end{tabular}

TABLE 3: Cuts of $\widetilde{b}_{k t}$.

\begin{tabular}{lccccc}
\hline$\widetilde{b}_{k t}$ & $k=1$ & $k=2$ & $k=3$ & $k=4$ & $k=5$ \\
\hline$t=1$ & $(3,6,9)$ & $(9,12,15)$ & $(11,14,17)$ & $(5,8,11)$ & $(7,10,13)$ \\
$t=2$ & $(5,8,11)$ & $(7,10,13)$ & $(13,16,19)$ & $(3,6,9)$ & $(9,12,15)$ \\
\hline
\end{tabular}

is a deterministic equivalent formulation of Model (2), where $\lambda \in[0,1], \alpha \in(0,1]$, and $\beta \in(0,1)$ are chosen large enough and

$$
\begin{aligned}
& f^{-}=\sum_{i=1}^{I} \sum_{t=1}^{T}\left[r_{i t}\left(d_{i t}^{-}-u_{i t}\right)-c p_{i t} p_{i t}-c q_{i t} q_{i t}-c u_{i t} u_{i t}\right], \\
& f^{+}=\sum_{i=1}^{I} \sum_{t=1}^{T}\left[r_{i t}\left(d_{i t}^{+}-u_{i t}\right)-c p_{i t} p_{i t}-c q_{i t} q_{i t}-c u_{i t} u_{i t}\right] .
\end{aligned}
$$

It is easy to see that Theorem 7 presents a method to find a robust optimal solution for the uncertain optimization Model (2).

\section{Case Study and Sensitivity on the Parameters of Flexibility Degree}

In this section, we shall apply the model and the method to solve a problem of production planning.

Suppose that there are 5 items, 5 resources, and 2 periods. The values of the parameters in Model (2) are listed in Table 1.

In this setting, by Theorem 7 , a deterministic equivalent formulation of Model (2) is obtained. Actually, the first group of constraints in (26) is equivalent to the following ten inequalities for $k=1,2, \ldots, 5$ and $t=1,2$, respectively:

$$
\begin{aligned}
& 0.5\left[(6-3 \alpha) p_{11}+(7-3 \alpha) p_{21}\right. \\
& \quad+(9-3 \alpha) p_{31}+(6-3 \alpha) p_{41} \\
& \left.\quad+(7-3 \alpha) p_{51}-(3+3 \alpha)\right]^{2}
\end{aligned}
$$

$$
\begin{aligned}
& -(1-\beta)(6-6 \alpha)\left(p_{11}+p_{21}+p_{31}+p_{41}+p_{51}\right) \\
& \times(6-6 \alpha) \\
& \leq 0 \\
& 0.5\left[(6-3 \alpha) p_{12}+(7-3 \alpha) p_{22}+(9-3 \alpha) p_{32}\right. \\
& \left.+(6-3 \alpha) p_{42}+(7-3 \alpha) p_{52}-(5+3 \alpha)\right]^{2} \\
& -(1-\beta)(6-6 \alpha)\left(p_{12}+p_{22}+p_{32}+p_{42}+p_{52}\right)(6-6 \alpha) \\
& \leq 0 \text {, } \\
& 0.5\left[(9-3 \alpha) p_{11}+(8-3 \alpha) p_{21}+(11-3 \alpha) p_{31}\right. \\
& \left.+(9-3 \alpha) p_{41}+(8-3 \alpha) p_{51}-(9+3 \alpha)\right]^{2} \\
& -(1-\beta)(6-6 \alpha)\left(p_{11}+p_{21}+p_{31}+p_{41}+p_{51}\right)(6-6 \alpha) \\
& \leq 0 \text {, } \\
& 0.5\left[(9-3 \alpha) p_{12}+(8-3 \alpha) p_{22}+(11-3 \alpha) p_{32}\right. \\
& \left.+(9-3 \alpha) p_{42}+(8-3 \alpha) p_{52}-(7+3 \alpha)\right]^{2} \\
& -(1-\beta)(6-6 \alpha)\left(p_{12}+p_{22}+p_{32}+p_{42}+p_{52}\right)(6-6 \alpha) \\
& \leq 0 \text {, } \\
& 0.5\left[(7-3 \alpha) p_{11}+(11-3 \alpha) p_{21}+(13-3 \alpha) p_{31}\right. \\
& \left.+(7-3 \alpha) p_{41}+(11-3 \alpha) p_{51}-(11+3 \alpha)\right]^{2} \\
& -(1-\beta)(6-6 \alpha)\left(p_{11}+p_{21}+p_{31}+p_{41}+p_{51}\right)(6-6 \alpha) \\
& \leq 0 \text {, } \\
& 0.5\left[(7-3 \alpha) p_{12}+(11-\alpha) p_{22}+(13-3 \alpha) p_{32}\right. \\
& \left.+(7-3 \alpha) p_{42}+(11-3 \alpha) p_{52}-(13+3 \alpha)\right]^{2} \\
& -(1-\beta)(6-6 \alpha)\left(p_{12}+p_{22}+p_{32}+p_{42}+p_{52}\right)(6-6 \alpha) \\
& \leq 0 \text {, } \\
& 0.5\left[(6-3 \alpha) p_{11}+(7-3 \alpha) p_{21}+(9-3 \alpha) p_{31}\right. \\
& \left.+(6-3 \alpha) p_{41}+(7-3 \alpha) p_{51}-(5+3 \alpha)\right]^{2} \\
& (1-\beta)(6-6 \alpha)\left(p_{11}+p_{21}+p_{31}+p_{41}+p_{51}\right)(6-6 \alpha) \\
& \leq 0 \text {, } \\
& 0.5\left[(6-3 \alpha) p_{12}+(7-3 \alpha) p_{22}+(9-3 \alpha) p_{32}\right. \\
& \left.+(6-3 \alpha) p_{42}+(7-3 \alpha) p_{52}-(3+3 \alpha)\right]^{2} \\
& -(1-\beta)(6-6 \alpha)\left(p_{12}+p_{22}+p_{32}+p_{42}+p_{52}\right)(6-6 \alpha) \\
& \leq 0 \text {, } \\
& 0.5\left[(9-3 \alpha) p_{11}+(8-3 \alpha) p_{21}+(11-3 \alpha) p_{31}\right. \\
& \left.+(9-3 \alpha) p_{41}+(8-3 \alpha) p_{51}-(7+3 \alpha)\right]^{2}
\end{aligned}
$$


TABLE 4: Optimal solution for $\lambda=0.75, \alpha=0.8$, and $\beta=\mathbf{0 . 9 / 0 . 8 5 / 0 . 8}$.

Optimal values of the objective function $f=\mathbf{1 7 0 . 0 3 2 2 / 1 7 0 . 0 2 8 5 / 1 7 0 . 0 2 4 6}$

\begin{tabular}{lrr}
$p_{i t}$ & \multicolumn{1}{c}{$q_{i t}$} & $u_{i t}$ \\
\hline$p_{11}=\mathbf{0 . 1 7 1 1 8 7 0 / 0 . 2 3 6 8 1 2 3 / 0 . 2 3 4 0 5 5 6}$ & $q_{11}=2.0 / 2.0 / 2.0$ & $u_{11}=0.0 / 0.0 / 0.0$ \\
$p_{12}=0.0 / 0.0 / 0.0$ & $q_{12}=0.0 / 0.0 / 0.0$ & $u_{12}=0.0 / 0.0 / 0.0$ \\
$p_{21}=0.0 / 0.0 / 0.0$ & $q_{21}=4.980137 / 4.980137 / 4.980137$ & $u_{21}=0.0 / 0.0 / 0.0$ \\
$p_{22}=0.1986301 / 0.1986301 / 0.1986301$ & $q_{22}=0.0 / 0.0 / 0.0$ & $u_{22}=0.0 / 0.0 / 0.0$ \\
$p_{31}=0.0 / 0.0 / 0.0$ & $q_{31}=4.0 / 4.0$ & $u_{31}=0.0 / 0.0 / 0.0$ \\
$p_{32}=0.4138537 / 0.0 / 0.0$ & $q_{32}=0.0 / 0.0 / 0.0$ & $u_{32}=0.0 / 0.0 / 0.0$ \\
$p_{41}=\mathbf{0 . 7 6 6 3 9 1 1 / 0 . 1 1 0 1 3 8 2 / 0 . 1 3 7 7 0 5 4}$ & $q_{41}=2.0 / 2.0 / 2.0$ & $u_{41}=0.0 / 0.0 / 0.0$ \\
$p_{42}=0.0 / 0.0 / 0.0$ & $q_{42}=0.0 / 0.0 / 0.0$ & $u_{42}=0.0 / 0.0 / 0.0$ \\
$p_{51}=0.0 / 0.0 / 0.0$ & $q_{51}=\mathbf{4 . 8 7 9 8 4 8 / 4 . 7 6 6 9 4 6 / 4 . 6 8 2 6 4 0}$ & $u_{51}=0.0 / 0.0 / 0.0$ \\
$p_{52}=\mathbf{0 . 1 2 0 1 5 2 3 / 0 . 2 3 3 0 5 4 4 / 0 . 3 1 7 3 6 0 1}$ & $q_{52}=0.0 / 0.0 / 0.0$ & $u_{52}=0.0 / 0.0 / 0.0$ \\
\hline
\end{tabular}

Bold values show the notable features of the parameter choices or the numerical results.

TABLE 5: Optimal solution for $\lambda=\mathbf{0 . 5} / \mathbf{0 . 2 5}, \alpha=0.8$, and $\beta=0.9$.

Optimal values of the objective function $f=\mathbf{2 7 4 . 0 3 2 2} / \mathbf{3 7 8 . 0 3 2 2}$

\begin{tabular}{lcr}
$p_{i t}$ & \multicolumn{1}{c}{$q_{i t}$} & $u_{i t}$ \\
\hline$p_{11}=0.1711870 / 0.1711870$ & $q_{11}=2.0 / 2.0$ & $u_{11}=0.0 / 0.0$ \\
$p_{12}=0.0 / 0.0$ & $q_{12}=0.0 / 0.0$ & $u_{12}=0.0 / 0.0$ \\
$p_{21}=0.0 / 0.0$ & $q_{21}=4.980137 / 4.980137$ & $u_{21}=0.0 / 0.0$ \\
$p_{22}=0.1986301 / 0.1986301$ & $q_{22}=0.0 / 0.0$ & $u_{22}=0.0 / 0.0$ \\
$p_{31}=0.0 / 0.0$ & $q_{31}=3.958614 / 3.958614$ & $u_{31}=0.0 / 0.0$ \\
$p_{32}=0.4138555 / 0.4138555$ & $q_{32}=0.0 / 0.0$ & $u_{32}=0.0 / 0.0$ \\
$p_{41}=0.7663911 / 0.7663911$ & $q_{41}=2.0 / 2.0$ & $u_{41}=0.0 / 0.0$ \\
$p_{42}=0.0 / 0.0$ & $q_{42}=0.0 / 0.0$ & $u_{42}=0.0 / 0.0$ \\
$p_{51}=0.0 / 0.0$ & $q_{51}=4.879848 / 4.879848$ & $u_{51}=0.0 / 0.0$ \\
$p_{52}=0.1201520 / 0.1201520$ & $q_{52}=0.0 / 0.0$ & $u_{52}=0.0 / 0.0$ \\
\hline
\end{tabular}

Bold values show the notable features of the parameter choices or the numerical results.

$$
\begin{aligned}
& (1-\beta)(6-6 \alpha)\left(p_{11}+p_{21}+p_{31}+p_{41}+p_{51}\right)(6-6 \alpha) \\
& \leq 0 \\
0.5 & {\left[(9-3 \alpha) p_{12}+(8-\alpha) p_{22}+(11-3 \alpha) p_{32}\right.} \\
& \left.\quad+(9-3 \alpha) p_{42}+(8-3 \alpha) p_{52}-(9+3 \alpha)\right]^{2} \\
& -(1-\beta)(6-6 \alpha)\left(p_{12}+p_{22}+p_{32}+p_{42}+p_{52}\right)(6-6 \alpha) \\
& \leq 0 .
\end{aligned}
$$

The second group of constraints is corresponding to the second part of inequalities in (26) with $k=1,2, \ldots, 5$ and $t=1,2$. It reads

$$
\begin{aligned}
& 3 \alpha p_{11}+(1+3 \alpha) p_{21}+(3+3 \alpha) p_{31}+3 \alpha p_{41} \\
& \quad+(1+3 \alpha) p_{51} \leq 3+3 \alpha \\
& 3 \alpha p_{12}+(1+3 \alpha) p_{22}+(3+3 \alpha) p_{32}+3 \alpha p_{42} \\
& \quad+(1+3 \alpha) p_{52} \leq 5+3 \alpha, \\
& (3+3 \alpha) p_{11}+(2+3 \alpha) p_{21}+(5+3 \alpha) p_{31}+(3+3 \alpha) p_{41} \\
& \quad+(2+3 \alpha) p_{51} \leq 9+3 \alpha,
\end{aligned}
$$

$$
\begin{aligned}
& (3+3 \alpha) p_{12}+(2+3 \alpha) p_{22}+(5+3 \alpha) p_{32}+(3+3 \alpha) p_{42} \\
& \quad+(2+3 \alpha) p_{52} \leq 7+3 \alpha \\
& (1+3 \alpha) p_{11}+(5+3 \alpha) p_{21}+(7+3 \alpha) p_{31}+(1+3 \alpha) p_{41} \\
& \quad+(5+3 \alpha) p_{51} \leq 11+3 \alpha, \\
& (1+3 \alpha) p_{12}+(5+3 \alpha) p_{22}+(7+3 \alpha) p_{32}+(1+3 \alpha) p_{42} \\
& \quad+(5+3 \alpha) p_{52} \leq 13+3 \alpha, \\
& 3 \alpha p_{11}+(1+3 \alpha) p_{21}+(3+3 \alpha) p_{31}+3 \alpha p_{41} \\
& \quad+(1+3 \alpha) p_{51} \leq 5+3 \alpha, \\
& 3 \alpha p_{12}+(1+3 \alpha) p_{22}+(3+3 \alpha) p_{32}+3 \alpha p_{42} \\
& \quad+(1+3 \alpha) p_{52} \leq 3+3 \alpha, \\
& (3+3 \alpha) p_{11}+(2+3 \alpha) p_{21}+(5+3 \alpha) p_{31} \\
& \quad+(3+3 \alpha) p_{41}+(2+3 \alpha) p_{51} \leq 7+3 \alpha, \\
& (3+3 \alpha) p_{12}+(2+3 \alpha) p_{22}+(5+3 \alpha) p_{32} \\
& \quad+(3+3 \alpha) p_{42}+(2+3 \alpha) p_{52} \leq 9+3 \alpha .
\end{aligned}
$$


TABLE 6: Optimal solution for $\lambda=0.75, \alpha=\mathbf{0 . 8 5} / \mathbf{0 . 9}$, and $\beta=0.9$.

Optimal values of the objective function $f=\mathbf{1 7 0 . 0 0 3 5} / \mathbf{1 6 9 . 9 7 4 1}$

\begin{tabular}{lcr}
$p_{i t}$ & $q_{i t}$ & $u_{i t}$ \\
\hline$p_{11}=\mathbf{0 . 2 6 2 4 1 4 5 / 0 . 1 0 6 2 2 5 5}$ & $q_{11}=2.0 / 2.0$ & $u_{11}=0.0 / 0.0$ \\
$p_{12}=0.0 / 0.0$ & $q_{12}=0.0 / 0.0$ & $u_{12}=0.0 / 0.0$ \\
$p_{21}=0.0 / 0.0$ & $q_{21}=\mathbf{4 . 8 6 8 4 6 5 / 4 . 8 8 6 8 3 8}$ & $u_{21}=0.0 / 0.0$ \\
$p_{22}=\mathbf{0 . 1 3 1 5 3 4 7 / 0 . 1 1 3 1 6 2 3}$ & $q_{22}=0.0 / 0.0$ & $u_{22}=0.0 / 0.0$ \\
$p_{31}=0.0 / 0.0$ & $q_{31}=\mathbf{3 . 9 4 8 1 5 0 / 3 . 9 3 7 6 3 3}$ & $u_{31}=0.0 / 0.0$ \\
$p_{32}=\mathbf{0 . 5 1 8 5 0 3 9 / 0 . 0}$ & $q_{32}=0.0 / 0.0$ & $u_{32}=0.0 / 0.0$ \\
$p_{41}=\mathbf{0 . 2 3 3 3 0 9 1 / 0 . 1 6 5 8 6 7 5}$ & $q_{41}=2.0 / 2.0$ & $u_{41}=0.0 / 0.0$ \\
$p_{42}=0.0 / 0.0$ & $q_{42}=0.0 / 0.0$ & $u_{42}=0.0 / 0.0$ \\
$p_{51}=0.0 / 0.0$ & $q_{51}=\mathbf{5 . 0 0 0 0 0 0 / 4 . 9 9 0 0 7 6}$ & $u_{51}=0.0 / 0.0$ \\
$p_{52}=\mathbf{0 . 0} / \mathbf{0 . 9 9 2 3 6 6 3}$ & $q_{52}=0.0 / 0.0$ & $u_{52}=0.0 / 0.0$ \\
\hline
\end{tabular}

Bold values show the notable features of the parameter choices or the numerical results.

Similarly, the third group of constraints in (26) is expressed as

$$
\begin{aligned}
& (6-3 \alpha) p_{11}+(7-3 \alpha) p_{21}+(9-3 \alpha) p_{31}+(6-3 \alpha) p_{41} \\
& +(7-3 \alpha) p_{51} \geq 3+3 \alpha, \\
& (6-3 \alpha) p_{12}+(7-3 \alpha) p_{22}+(9-3 \alpha) p_{32}+(6-3 \alpha) p_{42} \\
& +(7-3 \alpha) p_{52} \geq 5+3 \alpha, \\
& (9-3 \alpha) p_{11}+(8-3 \alpha) p_{21}+(11-3 \alpha) p_{31}+(9-3 \alpha) p_{41} \\
& +(8-3 \alpha) p_{51} \geq 9+3 \alpha, \\
& (9-3 \alpha) p_{12}+(8-3 \alpha) p_{22}+(11-3 \alpha) p_{32}+(9-3 \alpha) p_{42} \\
& +(8-3 \alpha) p_{52} \geq 7+3 \alpha, \\
& (7-3 \alpha) p_{11}+(11-3 \alpha) p_{21}+(13-3 \alpha) p_{31}+(7-3 \alpha) p_{41} \\
& +(11-3 \alpha) p_{51} \geq 11+3 \alpha \\
& (7-3 \alpha) p_{12}+(11-3 \alpha) p_{22}+(13-3 \alpha) p_{32}+(7-3 \alpha) p_{42} \\
& +(11-3 \alpha) p_{52} \geq 13+3 \alpha, \\
& (6-3 \alpha) p_{11}+(7-3 \alpha) p_{21}+(9-3 \alpha) p_{31}+(6-3 \alpha) p_{41} \\
& +(7-3 \alpha) p_{51} \geq 5+3 \alpha, \\
& (6-3 \alpha) p_{12}+(7-3 \alpha) p_{22}+(9-3 \alpha) p_{32}+(6-3 \alpha) p_{42} \\
& +(7-3 \alpha) p_{52} \geq 3+3 \alpha, \\
& (9-3 \alpha) p_{11}+(8-3 \alpha) p_{21}+(11-3 \alpha) p_{31}+(9-3 \alpha) p_{41} \\
& +(7-3 \alpha) p_{51} \geq 7+3 \alpha, \\
& (9-3 \alpha) p_{12}+(8-3 \alpha) p_{22}+(11-3 \alpha) p_{32}+(9-3 \alpha) p_{42} \\
& +(7-3 \alpha) p_{52} \geq 9+3 \alpha \text {. }
\end{aligned}
$$

The last group, associated with the fourth constraint in (26), is specified by

$$
\begin{aligned}
& (6-3 \alpha) p_{11}+(7-3 \alpha) p_{21}+(9-3 \alpha) p_{31}+(6-3 \alpha) p_{41} \\
& \quad+(7-3 \alpha) p_{51} \leq 9-3 \alpha
\end{aligned}
$$

$$
\begin{aligned}
& (6-3 \alpha) p_{12}+(7-3 \alpha) p_{22}+(9-3 \alpha) p_{32}+(6-3 \alpha) p_{42} \\
& +(7-3 \alpha) p_{52} \leq 11-3 \alpha, \\
& (9-3 \alpha) p_{11}+(8-3 \alpha) p_{21}+(11-3 \alpha) p_{31}+(9-3 \alpha) p_{41} \\
& +(8-3 \alpha) p_{51} \leq 15-3 \alpha, \\
& (9-3 \alpha) p_{12}+(8-3 \alpha) p_{22}+(11-3 \alpha) p_{32}+(9-3 \alpha) p_{42} \\
& +(8-3 \alpha) p_{52} \leq 13-3 \alpha, \\
& (7-3 \alpha) p_{11}+(11-3 \alpha) p_{21}+(13-3 \alpha) p_{31}+(7-3 \alpha) p_{41} \\
& +(11-3 \alpha) p_{51} \leq 17-3 \alpha, \\
& (7-3 \alpha) p_{12}+(11-3 \alpha) p_{22}+(13-3 \alpha) p_{32}+(7-3 \alpha) p_{42} \\
& +(11-3 \alpha) p_{52} \leq 19-3 \alpha, \\
& (6-3 \alpha) p_{11}+(7-3 \alpha) p_{21}+(9-3 \alpha) p_{31}+(6-3 \alpha) p_{41} \\
& +(7-3 \alpha) p_{51} \leq 11-3 \alpha, \\
& (6-3 \alpha) p_{12}+(7-3 \alpha) p_{22}+(9-3 \alpha) p_{32}+(6-3 \alpha) p_{42} \\
& +(7-3 \alpha) p_{52} \leq 9-3 \alpha, \\
& (9-3 \alpha) p_{11}+(8-3 \alpha) p_{21}+(11-3 \alpha) p_{31}+(9-3 \alpha) p_{41} \\
& +(8-3 \alpha) p_{51} \leq 13-3 \alpha, \\
& (9-3 \alpha) p_{12}+(8-3 \alpha) p_{22}+(11-3 \alpha) p_{32}+(9-3 \alpha) p_{42} \\
& +(8-3 \alpha) p_{52} \leq 15-3 \alpha \text {. }
\end{aligned}
$$

Thus, Model (26) is written as

$$
\begin{aligned}
\max f= & 520-416 \lambda-p_{11}-q_{11} \\
& -6 u_{11}-2 p_{12}-2 q_{12}-6 u_{12} \\
& -3 p_{21}-3 q_{21}-p_{22} \\
& -q_{22}-6 u_{22}-2 p_{31}-q_{31}-6 u_{31}-p_{32}
\end{aligned}
$$




$$
\begin{aligned}
& -q_{32}-5 u_{32}-p_{41}-q_{41}-6 u_{41}-2 p_{42} \\
& -2 q_{42}-6 u_{42}-3 p_{51}-3 q_{51}-p_{52}-q_{52}-6 u_{52}
\end{aligned}
$$

s.t. $(28)-(31)$,

$$
\begin{aligned}
& q_{11}+p_{12}+u_{12} \geq 2, \\
& q_{21}+p_{22}+u_{22} \geq 5, \\
& q_{31}+p_{32}+u_{32} \geq 4, \\
& q_{41}+p_{42}+u_{42} \geq 2, \\
& q_{51}+p_{52}+u_{52} \geq 5, \\
& q_{11}+p_{12}+u_{12} \leq 14, \\
& q_{21}+p_{22}+u_{22} \leq 16, \\
& q_{31}+p_{32}+u_{32} \leq 18, \\
& q_{41}+p_{42}+u_{42} \leq 14, \\
& q_{51}+p_{52}+u_{52} \leq 16, \\
& p_{i t}, q_{i t}, u_{i t} \geq 0, \quad i=1,2,3,4,5, t=1,2 .
\end{aligned}
$$

Take $\lambda=0.75, \alpha=0.8$, and $\beta=0.9$. Tables 2 and 3 present the cuts of the triangle fuzzy subsets $\widetilde{a}_{i k}$ and $\widetilde{b}_{k t}$, respectively.

With these coefficients shown in Tables 2 and 3, we can obtain an approximate optimal solution of Model (2) by solving problem (32). The numerical results are reported in Table 4 .

Next, we are interested in the sensitivity of the obtained optimal solution on the three parameters of flexibility degree, $\lambda, \alpha$, and $\beta$. By choosing different suitable values of $\lambda, \alpha$, and $\beta$, we check the changes of optimal solutions.

We first change the values of $\beta$ by fixing $\lambda$ and $\alpha$. The partial numerical results with $\beta=0.85$ and $\beta=0.8$ are reported in Table 4.

From Table 4, it is clear that the optimal value of the objective function increases with the increment of $\beta$. A part of the nonzero optimal solutions, such as $p_{41}, p_{52}$, and $q_{51}$, is seriously changed and another part of the nonzero optimal solutions appears to be more stable.

In Table 5, we report a part of numerical results with two different values of $\lambda$. It indicates that the optimal decisionmaking is basically stable except that the value of the objective function is different.

Finally, in Table 6, we report a part of numerical results with two different values of $\alpha$. It indicates that the choice of $\alpha$ greatly affects the optimal value of the objective function and the nonzero optimal solutions.

All of the results in Tables 4-6 demonstrate that the zero optimal solutions seem not to be sensitive on the change of the three parameters of flexibility degree $(\lambda, \alpha$, and $\beta)$. From these numerical results, it is concluded that the proposed solution method in this paper can find the relatively robust optimal solution for the original uncertain production planning problem.

\section{Final Remarks}

In this paper, a polymorphic uncertain linear programming (PULP) model for the generalized production planning problem was constructed where the uncertainty in the real production was taken into consideration.

For an optimization model involved with polymorphic uncertainty, it is impossible to find an optimal solution in the view point of standard optimization theory in general. In virtue of the method proposed in [12], we have found the approximate optimal solution of the generalized production planning problem by deriving the deterministic equivalent formulation.

Case study and numerical analysis have shown that the different parameters of flexibility degree had distinct sensitivity of optimal solution and the zero optimal solutions seem immune to the changing values of the flexibility degree parameters. From the numerical results, it is concluded that the proposed solution method can find the relatively robust optimal solution for the original uncertain production planning problem. Thus, it is helpful in the real-world decisionmaking.

\section{Conflict of Interests}

The authors declare that there is no conflict of interests regarding the publication of this paper.

\section{Acknowledgments}

The authors would like to express their thanks to the anonymous referees for their constructive comments on the paper, which have greatly improved its presentation. This research is supported by the Natural Science Foundation of Hunan Province (13JJ3002) and the National Natural Science Foundation of China (Grant nos. 71221061, 71071162).

\section{References}

[1] E.-H. Aghezzaf, C. Sitompul, and F. Van den Broecke, "A robust hierarchical production planning for a capacitated two-stage production system," Computers \& Industrial Engineering, vol. 60, no. 2, pp. 361-372, 2011.

[2] J. Mula, D. Peidro, and R. Poler, "The effectiveness of a fuzzy mathematical programming approach for supply chain production planning with fuzzy demand," International Journal of Production Economics, vol. 128, no. 1, pp. 136-143, 2010.

[3] S. A. Torabi, M. Ebadian, and R. Tanha, "Fuzzy hierarchical production planning (with a case study)," Fuzzy Sets and Systems, vol. 161, no. 11, pp. 1511-1529, 2010.

[4] R. Q. Zhang, L. K. Zhang, Y. Y. Xiao, and I. Kaku, "The activitybased aggregate production planning with capacity expansion in manufacturing systems," Computers \& Industrial Engineering, vol. 62, no. 2, pp. 491-503, 2012.

[5] S. A. Abass, M. A. Gomaa, G. A. Elsharawy, and M. S. Elsaid, "Generalized production planning problem under interval uncertainty," Egyptian Informatics Journal, vol. 11, no. 1, pp. 2731, 2010. 
[6] L. X. Tang, P. Che, and J. Y. Liu, "A stochastic production planning problem with nonlinear cost," Computers \& Operations Research, vol. 39, no. 9, pp. 1977-1987, 2012.

[7] D. Kira, M. Kusy, and I. Rakita, "A stochastic linear programming approach to hierarchical production planning," Journal of the Operational Research Society, vol. 48, no. 2, pp. 207-211, 1997.

[8] Y. F. Lan, Y. K. Liu, and G. Sun, "An approximation-based approach for fuzzy multi-period production planning problem with credibility objective," Applied Mathematical Modelling, vol. 34, no. 11, pp. 3202-3215, 2010.

[9] H. Jung, "A fuzzy AHP-GP approach for integrated productionplanning considering manufacturing partners," Expert Systems with Applications, vol. 38, no. 5, pp. 5833-5840, 2011.

[10] J. Mula, R. Poler, J. P. García-Sabater, and F. C. Lario, "Models for production planning under uncertainty: a review," International Journal of Production Economics, vol. 103, no. 1, pp. 271-285, 2006.

[11] S. P. Sethi, H. Yan, H. Zhang, and Q. Zhang, "Optimal and hierarchical controls in dynamic stochastic manufacturing systems: a survey," Manufacturing \& Service Operations Management, vol. 4, no. 2, pp. 133-170, 2002.

[12] Z. Wan, S. J. Zhang, and K. L. Teo, "Two-step based sampling method for maximizing the capacity of V-belt driving in polymorphic uncertain environment," Proceedings of the Institution of Mechanical Engineers C, vol. 226, no. 1, pp. 177-191, 2012.

[13] Z. Wan, K. L. Teo, L. S. Kong, and C. H. Yang, "A class of mix design problems: formulation, solution methods and applications," The ANZIAM Journal, vol. 50, no. 4, pp. 455-474, 2009.

[14] Z. Wan, F. Z. Meng, A. Y. Hao, and Y. L. Wang, "Fuzzy and stochastic parameters-based prediction method for the components of alkali in the sintering process of aluminium," Fuzzy Systems and Mathematics, vol. 25, no. 3, pp. 163-167, 2011. 


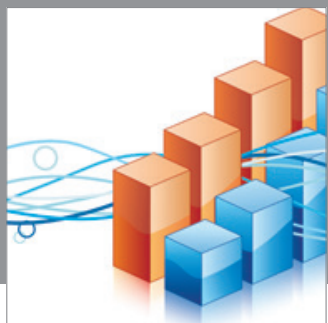

Advances in

Operations Research

mansans

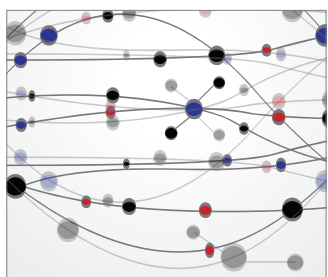

The Scientific World Journal
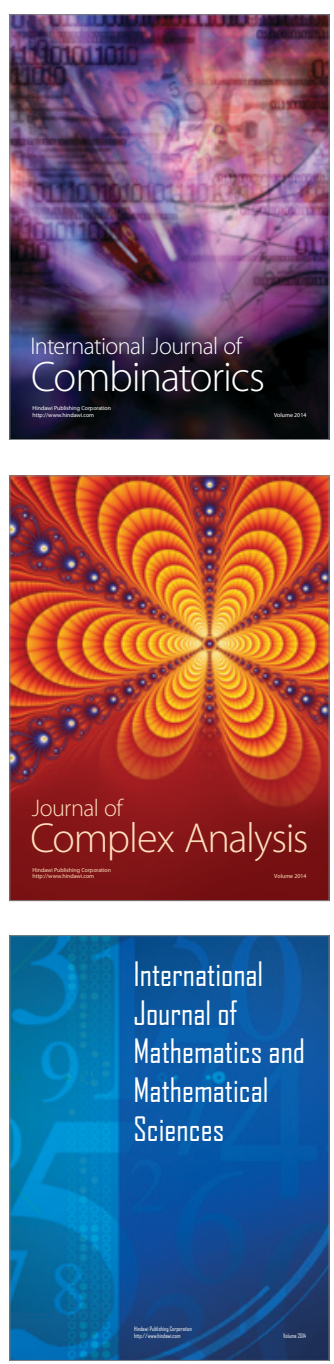
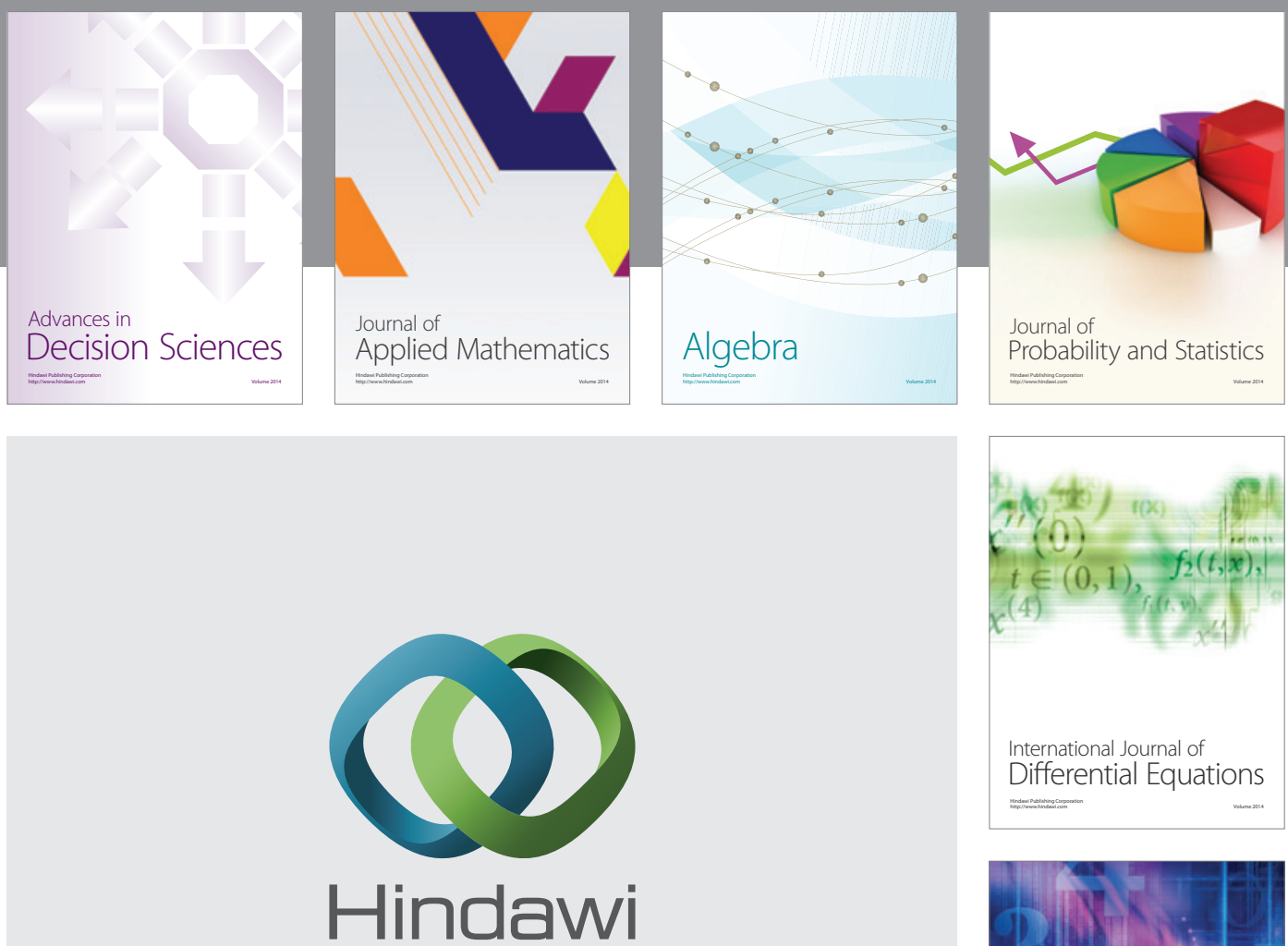

Submit your manuscripts at http://www.hindawi.com
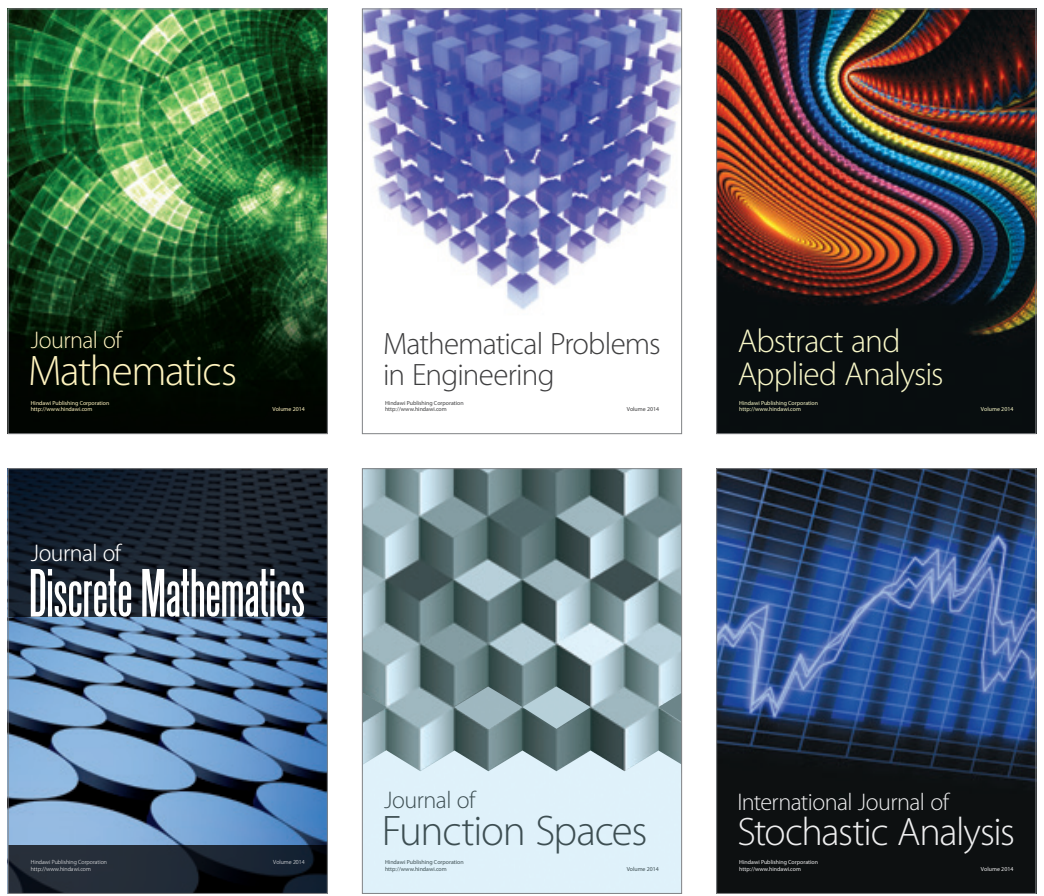

Journal of

Function Spaces

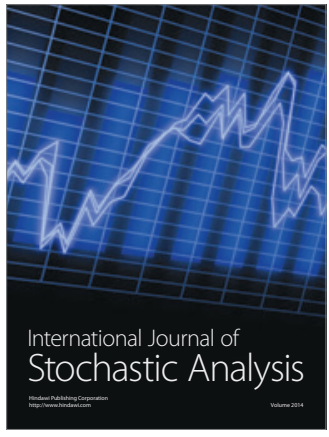

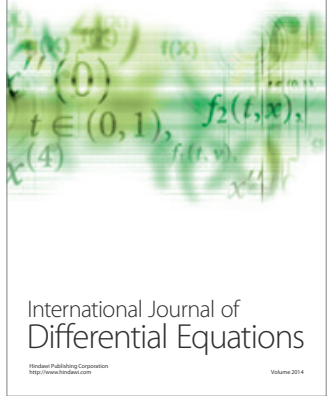
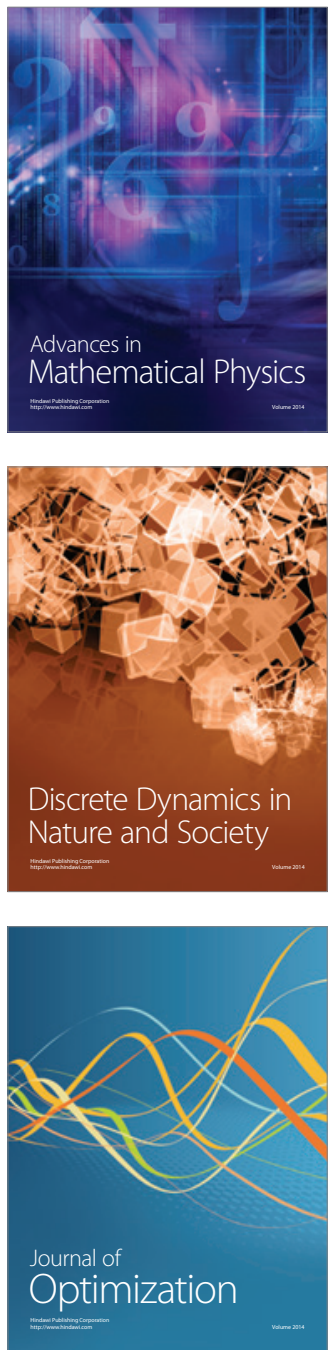